\title{
Attention Points of Research and Education in TCMP across Taiwan Strait and Recommendations for Future Research and Development of TCM in China Mainland Especially Fujian and Taiwan
}

\author{
Shengyan Xi ${ }^{1}$, Yanhui Wang ${ }^{1 *}$, Yaochen Chuang ${ }^{2}$, Linchao Qian, \\ Xiaoyan Qian ${ }^{1}$, Pengcheng $\mathrm{Li}^{1}$, Dawei Lu${ }^{1}$ \\ ${ }^{1}$ Department of Traditional Chinese Medicine of Medical College, Xiamen University, Xiamen, China \\ ${ }^{2}$ Center of General Education, Central Taiwan University of Science and Technology, Taichung, Taiwan \\ Email: ${ }^{2} 2076110 @ 126 . c o m$
}

Received April 5, 2013; revised April 22, 2013; accepted May 12, 2013

Copyright (C) 2013 Shengyan Xi et al. This is an open access article distributed under the Creative Commons Attribution License, which permits unrestricted use, distribution, and reproduction in any medium, provided the original work is properly cited.

\begin{abstract}
Objective: China mainland and Taiwan are separated by the Taiwan Strait, but their land edges are close to each other, blood relationship is very compact, and the origin is profound, the communication of Traditional Chinese Medicine (TCM) between China mainland especially Fujian and Taiwan district is more and more frequent. From the actuality and situation of traditional Chinese medicine and pharmacy (TCMP), the objective of this study was to briefly expound the points to which attention should be attached urgently in education, research and development of TCM between China mainland especially between Fujian and Taiwan, and be provide with several resolving threads and recommenddations to aim directly at the attention points, and wish it can offer some assistance to the development and generalization of the cross-Strait TCMP. Methods: The China Statistical Yearbook of Chinese Medicine (1987-2010), the Yearbook of Public Health of Taiwan (2009), the full-text data base of China National Knowledge Infrastructure (CNKI) (1993-2009), provides information on research and education in TCMP across Taiwan Strait in the last 10 years. The methods of analysis and comparison are applied in this study to show the TCMP situation between Taiwan and China mainland. Result: Due to the differences in history, district, policy and legislation, the TCMP's industry and trade, education, research and exploitation, standard and so on, have lots of differences between Taiwan and China mainland, and many barriers are produced in the communication and cooperation of cross-Strait TCM and pharmacy. Conclusion: China mainland especially Fujian and Taiwan have the fierce intention to carry out thorough investigation in many territories of TCMP. The prospects and development space of communication and cooperation of cross-Strait TCMP are quite broad.
\end{abstract}

Keywords: Traditional Chinese Medicine and Pharmacy (TCMP); Traditional Chinese Drug; Traditional Chinese Medicine (TCM); Chinese Herbal Medicine; Research and Development (R\&D); China Mainland; Fujian and Taiwan District; Recommendation

\section{Introduction}

Traditional Chinese medicine (TCM) is the splendid cultural treasure of the Chinese nation, and with long history and several thousand years of medication experience, it has accumulated a great deal of valuable materia medica data, which has an important guiding role on new medicine research and development (R\&D). Although with thousands of years of practice, Chinese herbal medicine

${ }^{*}$ Corresponding author. has formed its own unique and complete pharmacological system, due to the traditional route of administration and dosage form is more backward, effect shows slowly, and is difficult to standardize and normalize, especially the complicated ingredients in Chinese herbal medicine, the substantial basis of effect is not wholly clear, pharmacodynamic mechanism of action is not well known, and the content determination method is relatively rough. This greatly limits the industry development of Chinese medicine in China. In recent years, because of many de- 
ficiencies in the basic research of Chinese herbs, and unreasonable application, parts of the traditional Chinese drugs have shown some serious adverse drug reactions in clinic. The problem is highlighted particularly in the injections made by decoction pieces of traditional Chinese medicine in mainland, which is badly in need of new breakthrough [1]. Across the Taiwan Strait, the authority of Taiwan has constantly thought highly of the scientization of Chinese herbal drugs and the exploitation of Chinese Herbals [2], and the obvious developing advantages of Chinese herbal drugs of Taiwan, which comes from the good quality and the fine international image of the Chinese patent medicines produced in Taiwan [3]. Recently, the development of Chinese herbal industry in Taiwan district has been emphasized, and the research on traditional Chinese drug has been motivated to become the most activated branch in the domain of Taiwan present scientific research. Its research center is the new traditional Chinese drugs' exploitation. To surround this center, scientific researchers have carried out the items on exploiting for dried medicinal herbs, controlling on the quality of Chinese crude drugs, and studying their pharmacology [4]. We should thoroughly utilize this opportunity to positively strengthen the cooperation and communication on traditional Chinese medicine and pharmacy between China mainland and Taiwan district and to make up for each other's deficiencies, overcome the existing problems and share the benefits.

\section{Application and Trade of Chinese Herbal Medicine across Taiwan Strait}

Traditional Chinese medicine in Taiwan and Fujian has come from the same continuous Chinese culture, with distinct regional characteristics of Fujian province and Taiwan; traditional Chinese drugs have been widely used. About 66\% Taiwan populace usually apply traditional Chinese drugs. According to the investigation of Taiwan Pharmaceutical Manufacture's Association (TPMA), the most commonly used ten great Chinese crude drugs by Taiwan are Danggui (Radix Angelicae Sinensis), Shudihuang (Radix Rehmanniae preparata), Gancao (Radix Glycyrrhizae), Chuanxiong (Rhizoma Chanxiong), Fuling (Poria), Baishao (Radix Paeoniae Alba), Huangqi (Radix Astragali Mongolici), Baizhu (Rhizoma Atractylodis Macrocephalae), Renshen (Radix Ginseng), and Banxia (Rhizoma Pinelliae) respectively [5]. At present, more than 600 kinds of Chinese herbal medicines in Taiwan are dependent on imports, and of which 90\% come from the district of China mainland, and to some kinds of which, China mainland is the single source to import. In 2009, the export amount of mainland's Chinese crude drugs \& decoction pieces and extracts to Taiwan amounts to $98 \%$ of the total amount of exports of traditional Chinese drugs to Taiwan [3] (Figure 1). In 2010, Taiwan imported about 5000 batches of traditional Chinese medicinal materials, the gross weight of which was more than 13,000 tons, the market value of which was 5.5 hundred million Yuan, among which Hongzao (Fructus Jujubae), Huangqi (Radix Astragali Mongolici), Danggui (Radix Angelicae Sinensis) and Gancao (Radix Glycyrrhizae) count for nearly 4.0 hundred million Yuan. Throughout Taiwan, Chinese herbal medicine is also often used as health food material. Now more than 170 health food products in Taiwan have obtained their conformity certifications, among which 61 products has used Chinese herbal medicine as raw materials, the tea, ginseng, fungi and Hongqu (Ultivarietas Oryzae Sativae et Monasci) are the most, which occupied by $35.88 \%$ [6]. It is thus evident that the dependency of Taiwan on China mainland's traditional Chinese drugs is high.

\section{Research of Chinese Herbal Medicine and Compound Recipe in Taiwan District}

About the study of traditional Chinese medicine and pharmacy, since the 1970s, Taiwan authorities began opening up to the scientific research on Chinese herbal drugs. And the basic and clinical research on the disease that had a significant effect by TCM and the prescription that commonly used was carried out, which under the efficacy assessment of Chinese medicine. For example, Prof. Shih TB had researched the immunoregulation effects of Four-Ingredient Decoction (Siwu Tang), Tonifying the Middle and Replenishing Qi Decoction (Buzhong Yiqi Tang) and Pulse-Generating Powder (Shengmai San), who found that the three compound recipes had the different stimulating effects on many kinds of founder cells of hematopoietic community, and the protecting actions to the decrease of leucocytes caused by 5-Fluorouracil (5-FU) and could repair the hematopoiesis function [7]. The processing methods, quantitative analysis and safety testing to herbal medicines, have been in depth study. Prof. Wun WC has found that unprocessed Pinellia tuber or Ban Xia (Rhizoma Pinelliae) processed for seven days follow the incunabula had no toxicity in cell experiments or no acute lethal dose (LD) in rat experiments [8]. Prof.

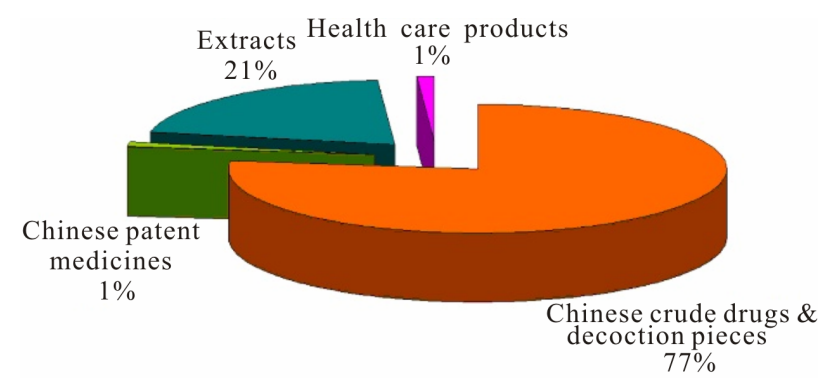

Figure 1. Product structure statistics of mainland's export amount of traditional Chinese drugs to Taiwan in 2009. 
Liou SS's research has shown that the content of Magnolol and Honokiol in Houpu (Cortex Magnoliae Officinalis) parched by ginger pop were respectively increased 5.6 and 4.1 times [9]. And it had been also continuously mixed with the modern scientific and technological achievements, and to explore the combination between Chinese medicine research and genomics research, and planning a "correlated research between TCM and genome" to develop the study of sthenic zheng of TCM [10]. In the modernization of traditional Chinese medicine research, the extraction, analysis and purification of Chinese herbal medicine are mainly in application. For example, the plant Jia Ci Shu (Casearia membranacea Hance) in Taiwan, Prof. Guh JH [11] has purified ten kinds clerodane diterpenoids from which have anticancer active, which all could effectively promote the apoptosis of tumor cells. It is worth mentioning that Taiwan has established the high-end platform of development and verification of Traditional Chinese Medicine, and by means of "Drug-similar" concept to find the drugs that are similar to the profiles of gene expression of Chinese herbal medicine to further understand the mechanism or effect of Chinese herbal medicine, may accelerate the opportunity that the effective Chinese herbal medicine or other drugs can enter the clinical trial [12]. And the obvious modern progress has been made in TCMP research of Taiwan.

\section{Higher Education of TCMP in Taiwan}

At present, the normal higher educational institutions of TCMP in Taiwan are mainly the two private universities, China Medical University (CMU) and Chang Gung University (CGU). The former has set up the college of TCM, school of Chinese medicine for eight years and school of post baccalaureate Chinese medicine for five years, and Master's and doctor degree programs of Graduate Institute of Chinese Medical Science, Master's degree program of Graduate Institute of Integrated Medicine (GIIM), and Master's degree program of Graduate Institute of Acupuncture Science. The latter only has the school of Chinese medicine for eight years and Master's degree program of Graduate Institute of Traditional Chinese Medicine [13] (Table 1). Except the CMU, the other schools are all relatively less scale of TCMP education. Concretely speaking, the education courses of Chinese medicine in Taiwan's higher schools are mainly as follows: The Graduate Institute of Natural Products of Chang Gung University, which has set up the "Pharmacology of TCM", "Information Research of Chinese Medicine”, etc.; China Medical University has the school of Chinese Pharmaceutical Sciences and Chinese Medicine Resources, and has cultivated the professional persons who mastered the resources of herbs, research and development of herbs by biotechnology, and management of herb resources. The master curriculums of CMU have "Monography of Herbalism”, "Special Discussion of Chinese Materia Medica”, "Industry Technology of Chinese Herbal Medicine”, etc. The doctor curriculums have "Special Topics of Herbalism”, "Special Topics of Chinese Pharmacy”, "Special Topics of Research Methods of Chinese Pharmacy”, etc. The Department of Pharmacy and Graduate Institute of Pharmaceutical Technology of Tajen University has set up the "Special Topics of Pharmacology of Traditional Chinese Drug”, "Special Topics of Pharmacological Identification”, "Pharmaco preparing Process of Traditional Chinese Drug”, "Special Topics of Materia Medica Formulas”, etc. The Biological Resources Department of Taipei Medical University has set up the "General Discussion of Traditional Medicine", "Herbalogy", "Medicated Diet and Food Therapy" and other courses [14]. We know that every hierarchical education of TCMP from the under graduate course to doctor curriculum has been opened in Taiwan now. But compared with China mainland's TCM courses that are complete in ranges, curriculum taxons of Taiwan TCM are not enough, and the contents' profundity and scope are also more inferior.

\section{Attention Points of Cross-Strait TCMP Research and Education Needed Urgently}

With the development of the economy, the society and culture exchanges between the mainland and Taiwan are more and more close. TCM that carries the history characteristics of the Chinese nation has taken the lead on cooperative research for standardization, normalization, and modernization of cross-Strait traditional Chinese

Table 1. Overview of Taiwan bachelor education of TCM.

\begin{tabular}{|c|c|c|c|c|c|}
\hline School Name & Department Name & $\begin{array}{l}\text { Study Time } \\
\text { Limit }\end{array}$ & $\begin{array}{l}\text { Established } \\
\text { Time }\end{array}$ & Annual Enrollment & $\begin{array}{c}\text { Number of } \\
\text { Graduate }\end{array}$ \\
\hline \multirow{2}{*}{ China Medical University } & \multirow{2}{*}{ School of Chinese Medicine } & Seven years & 1966-1995 & 120 & 2180 \\
\hline & & Eight years & 1996-up to now & 120 & 404 \\
\hline Chang Gung University & School of Chinese Medicine & Eight years & 1998-up to now & 50 & 81 \\
\hline China Medical University & $\begin{array}{c}\text { School of Post Baccalaureate Chinese } \\
\text { Medicine }\end{array}$ & Five years & 1984-up to now & 100 & 1449 \\
\hline
\end{tabular}

Notes: Data from Public Health Reports (2005) and Chinese Medicine Administration Indicators (2007). 
drug with a series of positive and effective exploration. Through the constant exchange and joint research, it has made significant progress and formed a good development momentum. But there also both exist considerable problems that inheriting traditional characteristics of TCM and carrying forward to TCM or generalizing TCM are not enough, post marketing of traditional Chinese drug and quality requirements of Materia Medica are not strict, the groundbreaking of new traditional Chinese drug is insufficient, and the key technology breakthrough is difficult, and so on, especially in the development and research of Chinese crude drugs and decoction pieces.

\subsection{Analysis in Industry and Trade of TCMP}

In China mainland, the Chinese medicine agriculture has started for many years. Prof. Huang SL pointed out that the sustainable development and rational utilization of traditional Chinese drug resources, and related ecological or environmental problems were not resolved effectively; some Chinese drug industrial arts and engineering technologies in some industries were relatively backward; the production efficiency and utilization capacity were relatively low; some industries lack standardized and tailor-made pharmaceutical industry equipments [15]. From the above-mentioned application of Chinese herbal drugs of Taiwan and the cross-Strait import or export amount of Chinese crude drugs, we know that the Taiwan industry of Chinese medicine has faced many problems, such as few natural resources of Chinese medicinal materials, Materia Medica depending on China Mainland extremely, small scale of Chinese patent medicine industry, difficult exploitation of fire new patent medicine, and so on. To obtain Chinese medicine Taiwan needs to import it. As for the Chinese crude drugs, they are quite abundant in Mainland, but the ratio of Taiwan importing the health care products and Chinese patent medicines from China Mainland is tiny [16]. And in order to enhance the competitiveness, since 2009, “the Executive Yuan” of Taiwan has listed Chinese herbal medicine industry as one of the Taiwan's major development projects, strengthened infrastructure and improved the industrial environment to impulse the market development [17]. In China Mainland, the situation about the exports of Chinese medicine mainly depends on the bulk drugs and the old species has no significant change [15]. Therefore, there exist strong complementarities of cross-Strait Chinese medicine industry and trade, and there will be more cooperation items.

\subsection{Analysis in Education and Elite Cultivating in TCMP}

Today's main international medicine is the modern western medicine, the economy income and social status of traditional profession doctor is not better than western medicine doctor's, especially in Taiwan. The phenomenon of westernizing tendency in TCMP is the best annotation for this embarrassing reality. Prof. Su XY [14] pointed out that all the mode of education and training of traditional Chinese medicine and pharmacy in Taiwan trends to westernizing for its high proportion western medical courses, and higher education of traditional Chinese drug usually ignore the "patient-oriented pharmaceutical care education”. For instance, the purpose of running China Medical University is to "unify the Chinese and western medicine to establish new medical science", and to emphasize the important position of western medicine in TCMP education. Currently, except the westernizing tendency, there are still lots of problems in the education of TCMP in Taiwan that need to be resolved urgently, such as that the teaching faculty of TCMP is quite insufficient, the teachers who have abundant clinical experience but less teaching experience, the deficient teaching materials of TCMP that unitively compiled by Taiwan natives, and few clinical teaching hospitals of TCM [13]. The supply of teachers in School of Chinese Medicine of Chang Gung University is mostly the visiting professors who are invited from TCM colleges or universities in China mainland, their TCM teaching materials are the fifth edition teaching materials of the mainland high medicine schools; but CMU mostly uses the self-compiled teaching materials [18]. Prof. Shen JZ points out that the teaching materials applied by school of Chinese medicine and post baccalaureate Chinese medicine are out of date to cause some learning perplexity to students, that mainly because the materials had no unity and no systematicness, and been compiled too early [19]. Except this problem, there are few magazines about TCMP in Taiwan, just several kinds like "Journal of Chinese Medicine”, "Taiwan Clinical Journal of Traditional Chinese Medicine”. Nowadays, there are no departments of Chinese traditional medicine set up in Taiwan's public hospitals, and its medical service laws restrict the traditional Chinese physicians to use western medicines, so the self-sustaining traditional Chinese hospitals provide little TCM service to in-patients for the deficiency in emergency treatment assisting to patients by western medicine, and furthermore, no health insurance has been internalized to the in-patients of TCM hospitals, and the clinic beds of TCM are less [20]. The Quantity of Traditional Chinese Medical Hospital in China Mainland are increasing steadily from 2141 hospitals in 1990 to 3232 hospitals in 2010 [21]; but the Quantity of TCM Hospital in Taiwan was in decrease year by year from 109 hospitals in 1994 to 22 hospitals in 2008 [22], see Figure 2. And as a result, the clinical education and development of TCMP needs urgent focus by the Taiwan authority. To touch upon the medical 


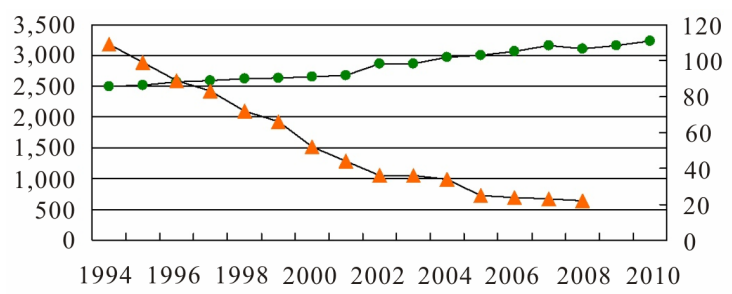

$\rightarrow$ Quantity of Traditional Chinese Medical Hospital in China Mainland -t- Quantity of Traditional Chinese Medical Hospital in Taiwan District

Figure 2. Comparison of quantity of Traditional Chinese Medical Hospital between China mainland and Taiwan District.

education pattern in Taiwan from the annual enrollment, discipline setup, curriculum or study time limit, it is certainly the Alma education. Generally speaking, the atmosphere and cultural heritage of TCMP education in Taiwan is not profound, and inferior to China mainland. But it is advisable to develop the TCM and Western medicine simultaneously, to thin the course offering, and emphasize the practice skills cultivation in Taiwan. By special examinations, people can obtain the qualifications of physicians and practitioners in Taiwan. Although the educational background of personnel participated in this special examination is increasing to some extent, most has low culture degree, high proportion personnel who passed the examination has no systemic and normal clinical skills training [18]. Furthermore, there is no examination system of licensed TCM pharmacists in Taiwan. The in-service education to Taiwan professional person in traditional Chinese drug, actually, is a remedied method to redeem the deficiency of pure TCM pharmacists in Taiwan, which aims at improving diathesis to pharmacists and practitioners of Chinese medicine, and belongs to irregular education of academic career. Just as a scholar pointed out that the cultivation of pure pharmacists of Chinese medicine in Taiwan needs to be achieved through subject differentiation and curriculum reform urgently [23]. In a word, in Taiwan District, the professional personnel for work or research engaged in traditional Chinese drug, the systematical studying about the basic theory of traditional Chinese drug, or professional training institutions are less, but the westernized phenomenon is quite serious. Taiwan TCM education has not yet formed the scale, and not still been brought into the formal trajectory. Lots of graduates possess the western medicine license and deal with western medicine or change to do something else. Therefore, the number of practitioners in TCMP is limited, elite mastered traditional Chinese medicine is lost.

\subsection{Analysis in Characteristics Maintaining to TCMP}

In China mainland, people in some developed coastal districts in economy such as Fujian, Guangdong usually trust the TCM relatively, but the supporting diagnostic facilities in Chinese medical institutions in part of mountain area of China mainland are in need of development. The characteristics and culture building of TCMP still need to be further strengthened by Chinese medical authorities. In these districts, taking advantages of characteristics and clinic techniques of TCMP to diagnose and treat, compared with the developed areas of TCMP, is not enough. In Taiwan, the phenomena also exist extensively. As the above-mentioned, the TCMP education and research is westernizing, the traditional Chinese medical hospitals are few. Especially in the effect study on Chinese crude drugs, compared with China mainland, more attention should be paid to purify the monomers to test their actions, not to the compatibility of traditional Chinese medicine prescriptions and the basic theory like drug-nature of Chinese medicine. Unfortunately, with the amount increasing of TCM hospitals in China mainland, the great problem is the westernizing in diagnosis and treatment [24]. Keeping the characteristics and culture of TCM becomes more and more difficult.

\subsection{Analysis in Research and Exploitation of Chinese Herbal Medicine or New Chinese Materia Medica Preparation}

Due to the historical reasons between Taiwan and China mainland, even if depending on their own advantages and painstaking research respectively, Chinese Herbal Medicine research in both Taiwan and China mainland still exist some insufficiency like the problems of the material basis clarification of perplexing mechanisms of action and of how to bring Chinese Herbal Medicine in line with modern science. The new drugs of TCM that have real curative effects in clinic and good economic benefits are seldom [25]. The reasons are multifold, such as emphasizing inadequately on prophase screening to effective prescriptions, pursuing the high and new preparation level and ignoring the potency of formula itself, falling short of standardization and normalization in clinical trials of Chinese Herbal Medicines, rare clinical revaluation to postmarketing new drugs, the shortage of basic research on drugs that only several hundred CHMs have been lucubrated in more than ten thousand kinds, having restricted the preparation development, not undertaking researches to aim directly at the market since the scientific research institutions and universities have the powerful strength on drug exploitation, and not closing up on the connection of the resources between the scientific research institutions, universities, and enterprises [25,26]. At present, Taiwan's Chinese materia medica preparations authorized by the Taiwan TCM Committee are all recorded in traditional ancient books and records, and the therapeutical indications all belong to the conceptual 
category of TCM. The new drug of TCM research in Taiwan is mainly involved in the anticancer, lowering blood pressure or blood fat, anti-osteoporosis, anti-gastrelcoma, relieving cough and antiasthma, treating stroke and melancholia, drug abstinence and so on [27]. Compared with the numerous scientific research institutions and multitude researchers, and strong support from Ministry of Public Health, Ministry of Science and Technology and State Administration of Traditional Chinese Medicine in China mainland, the research scope of Taiwan Chinese Herbal Medicine is narrower, and the number of Taiwan CHM research items is also less. Though the research of material basis and effect mechanism of some Taiwan idiomatical Chinese herbal medicines or minority ethnic drugs such as Niu Zhang Zhi (Antrodia Camphorata) [28,29] has been gradually carried out in China mainland, the cooperation and research in natural resources investigation, introduction and cultivation, and exploitation of cross-Strait idiomatical Chinese herbal medicines are not enough. The Chinese medicinal products with fine quality and high efficiency are still the chasing target of the TCM industry both in Taiwan District and China mainland. The capabilities in independent research, development, innovation and generalization of Chinese drugs in both sides across the Taiwan Strait are required to expand and accelerate. To develop the Chinese medicine products with cross-Strait common intellectual property rights is required urgently. Therefore, the cross-Strait thorough joint to research traditional Chinese drugs is imperative, meanwhile, has quite broad prospects and development space. Just as the researcher Huang Y [4] has pointed out that the future direction and priorities of traditional Chinese medicine research in Taiwan was still around the development of new Chinese drugs, and to undertake a series research of scientization, modernization and internationalization of traditional Chinese drugs.

\subsection{Analysis in Standard, Policy and Management in TCMP}

Taiwan and China mainland have been cut off from each other for more than 40 years, and development of TCMP between them has formed some differences. For example, some Chinese herbal medicines are not unified in species, such as Ji Xue Teng in China mainland is referred to the Spatholobus suberectus, but in Taiwan is referred to Millettia dielsiana, Mucuna birdwoodiana, or Mucuna sempervireus; and Fang Ji, Niu Xi, Shayuan Zi, Fang Feng, Xi Xin, Baijiang Cao, Kun Bu, also exist this phenomena [30]. These differences may be resulting in some obstacles for cooperation each other. The standards of preparation and application of traditional Chinese drugs are also not uniform. Both sides have such problems, so there are efforts should be made to develop appropriate legislation to reform and update. But the evaluation methods and standards system that are suitable for research of traditional Chinese medicine with its own characteristics has not been established [15]. The problems such as the criteria for determining the therapeutic effect of traditional Chinese medicines still restricted them to the international market; the exchanges and cooperation of TCM between China mainland and Taiwan are still restrained by the historical and geographical multifactor, cross-Strait policies and regulations, and so on. For example, Taiwan's traditional Chinese medicine research and application follows the "Taiwan Traditional Pharmacopoeia (TWTP)”, but in China mainland it follows the "Pharmacopoeia of the People's Republic of China (PPRC)", which some standards of CHM origin and pharmaceutics have the distinctions. Furthermore, there are many differences in the quality testing standards of cross-Strait traditional Chinese drugs [31]. The research and control to the active ingredient in China mainland is higher than in Taiwan, but Taiwan pays more attention to the control of the security index. To the Chinese crude drugs purchased from China mainland before the use, the test items such as the heavy metal contamination and pesticide residues are more, and the limited requirements are higher. Just as the chairman of TCM Committee of Department of Health of the Executive Yuan in Taiwan Huang LH [3] pointed out that "the current import of Chinese crude drugs was short of the all round borderland checkout mechanism, and between China mainland and Taiwan, the pharmacopoeia norm, test standard and management also had great differences. Meanwhile, the channel of the correlated experience and technical exchange was deficient, and there were no report windows for intercommunication and coordinating mechanism to be established for the disqualified Chinese crude drugs by the sampling inspection”.

\subsection{Analysis in Safety and Quality of Traditional Chinese Drugs}

Currently, to the Chinese herbal medicines, the Taiwan populaces mostly worry about that the heavy metals, pesticide residue, too many microorganisms and flavacin contained inside $[3,16]$. In China mainland, these problems also exist. Furthermore, the safety issues of traditional Chinese drug injections, especially the anaphylactic responses, are paid more and more close attention to [1]. In our opinions, the safety problems on the clinical application of some toxic Chinese herbal medicines or Chinese patent medicines especially containing the poisonous herbs or ingredients also need to be thought highly of. The processing or preparation quality of traditional Chinese drug should be put on the first place to some extent. 


\section{Recommendations for R\&D of TCMP between China Mainland Especially Fujian and Taiwan}

Fujian and Taiwan District, although facing each other across the Taiwan Strait, having the common ancestry and the same Fukienese, the similar customs and climate, but were isolated by the historical anthropogenic factors for a long time. Along with the alleviation of Cross-Strait Relations step by step, for the recent years, communication between China mainland especially the Fujian province and Taiwan District is increasing. It is quite frequent and direct more than the other provinces. The technology and culture exchange become active. Cross-Strait Relations get into a new period. And further understanding of the current situation between each other in science technology development, creating conditions to enhance the two districts' communication in medical science, has the far-reaching history and reality meaning with regard to promoting development of cross-Strait Medical treatment and health service, improving the health level of bilaterial compatriots, and multiplying and thriving of the whole Chinese nation [32]. These attention points, contained so many differences of cross-Strait, which need to be improved and overcome or resolved by the substantial investment of human, material and financial resources and time, may provide a good way for China mainland and Taiwan to get in touch with each other. It is the major subjects in cross-Strait TCM field to organize firstclass researchers and educators jointly by both sides, and to use the advanced R\&D and education platform, to adopt the advanced, reasonable methods and education cooperation for the development of traditional Chinese medicine and pharmacy.

\subsection{Strengthen the Cooperation of Industry and Trade of Traditional Chinese Drugs}

In order to resolve the obstacles in the cooperation of industry and trade of Chinese medicine, we think that the industrial platforms of cross-Strait traditional Chinese drug especially the one of the specific ethnic drugs in Fujian and Taiwan must be established first to exploit several Chinese medicine products with cross-Strait common intellectual property rights through utilizing each other's advanced experience. The pharmaceutical enterprises in both sides should consummate the pharmacectic flow sheet in common, penetrate each other deeply to be familiar with the respective custody laws and regulations of drugs and market demands, and avoid blindness in investment and market risk. Both sides should try their best to reduce the differences in legislation to improve the confidence in investment and cooperation. With regard to the trade of TCMP, due to the demand for body health care and diagnosis and treatment to disease, Taiwan District should enhance the import proportion of Chinese patent medicines and TCM health care products from China mainland. The cooperation of industry and trade of cross-Strait traditional Chinese drug is the future objective requirement, and also the necessity of history development.

\subsection{Strengthen the Education and Elite Cultivating in TCMP}

In view of the current situation of cross-Strait TCMP, we think that strengthening the education cooperation of cross-Strait traditional Chinese medicine and the cultivation of specialized elite who can master the related information of cross-Strait traditional Chinese medicine and pharmacy is quite necessary. Firstly, Taiwan District should organize experts to compile the unified teaching materials that are fit for the district characteristic and establish the complete supply system of teachers, and increase the TCMP curriculums in TCM discipline to change the tendency of over-emphasizing western medicine education, and expand the space and level of knowledge and academic exchange of cross-Strait Chinese herbal medicine, expand collection and sorting of the documents of traditional Chinese drugs in both sides, especially in Taiwan; and carry out the deep learning of ancient and modern Chinese medicine literature of Fukien and Taiwan with multi-disciplinary theoretical approaches and technologies of philosophy, social sciences, history, information science, computer technology and so on [33]. Secondly, Taiwan District should set up the regular examination system of traditional Chinese physicians and traditional Chinese pharmacists by drawing assistance from China mainland, and establish a joint cultivation system to cultivate the traditional Chinese pharmacist who can master the cross-Strait Chinese medicine, and allow the genuine pharmacists who know the clinical application and research of Chinese drugs to give full play the guiding role in these areas. Thirdly, Taiwan District should increase the number of TCM hospital and set up the department of TCM in public hospitals to give the TCM students more practice opportunities. Finally, Taiwan District may increase the kinds of magazines in TCMP to expand people's understanding of TCMP and strengthen the influence of TCM.

\subsection{Outstanding the Characteristics of TCMP}

How to keep the characteristics of traditional Chinese medicine in clinical practice and research is an important topic to the authority and TCMP workers both in Taiwan District and China mainland. We think that the urgent priority is to stop the westernizing of cultivation plan of TCM student in university or college, and take the cultivation model of the "disciple-following-teacher" conven- 
tion that imparted and inherited since ancient times according to the growth regularity of famous veteran teran doctors of TCM to cultivate TCM student. During the clinic process, the traditional Chinese physicians should try their best to make use of four diagnostic methods (inspection, listening and smelling, inquiry, palpation and pulse taking) to diagnose disease and CHMs, compound recipes or acupuncture to treat, especially in the TCM department of general hospitals. And in the R\&D of TCM, researchers must insist on the instruction of basic theory of TCM.

\subsection{Strengthen the Research and Exploitation of Chinese Herbal Medicine or New Compound Recipe}

Firstly, we think that it is urgent to strengthen the investigation of resources of the famous-region drugs in Taiwan and their introduction, breeding and developing in China mainland especially in Fujian province, including identifying the biogenesis of local Chinese herbal medicines that commonly used in Fujian and Taiwan, and summing up the nature and flavor, efficacy, usage, information about medicinal and edible dual purpose of the herbs. Relying on the advantages of wildlife resources, patterns implantation and medicinal industrial park of Fujian and Taiwan region, both sides should take the natural resources investigation, monitoring and regional planning to special local famous-region drugs, and establish gene banks of wild herbs and banks of germplasm resources, especially focus on carrying out the standardized planting of Chinese herbal medicines, the mutual introduction of cross-Strait herbs, and research and development of medicinal resources, and establish the introduction viewing area, the interzonal region test area, and demonstration area of good agriculture practice (GAP) stock breeding of cross-Strait famous-region drugs and endangered or bare drugs, and cultivate the modern raw material depot of traditional Chinese drugs to begin to take shape. Secondly, we think that both sides should aim directly at the Chinese herbal drugs that have cross-Strait national or local characteristics, and enlarge the pharmacy research. The focal point is to strengthen the research on therapeutic effect and mechanism of active components of idiomatical Chinese herbal medicines in China mainland especially Fujian and Taiwan. For example, the thorough research for drug-nature and channel entry, efficacy, and active constituents of Niu Zhang Zhi (Antrodia Camphorata), Xiao Ye Shan Pu Tao (Vitis Thunbergi), Jian Qu (Massa Medicata Fermentata), Feng Ju Dou Cao (Sarcopyramis Nepalensis Wall.), Ba Ji Tian (Morindae Officinalis How.), Tai Wan San Jian Shan (Cephalotaxus Wilsoniana Hayata.) [34] and so on, is very worthy to carry out. The research on the material basis, mechanism of action, clinical thera- peutic effect evaluation, and clinical safety evaluation of the cross-Strait new specific Chinese drugs or new compound preparations being used for the treatment to some common momentous diseases such as multiple tumor, diabetes, cardiovascular disease, neurogenic disease, hepatopathy and nephropathy in Fujian and Taiwan, also should be impelled actively. But the most important centric position of "therapeutic effect" should be established in the drug manufacturing. Thirdly, we think that both sides should choose the potential species to take the secondary thorough research and development from the existing idiomatical compound preparations in Fujian and Taiwan. China mainland especially Fujian and Taiwan may aim directly at the cross-Strait idiomatical traditional Chinese patent medicines and simple preparations, and use a variety of combinatorial chemical or biological methods to explain their scientific connotation, and exploit their upgrade and update Chinese herbal products. The Research Priorities are the differences in components, effects and toxicity between Taiwan's “Scientific Chinese medicine granule" and China mainland's “Traditional Chinese medicine preparation”, and promote the Taiwan's "Scientific Chinese medicine granule" to get into China mainland through Fujian province's R\&D platform. According to the standard of new drug approval of China mainland, Fujian pharmaceutical enterprises should combine with Taiwan's enterprises and scientific research units to proceed the clinical pharmaco-research of cross-Strait idiomatical new drugs, which is the Clinical Trials and Clinical Verification of new drug, and hasten the West Bank of Taiwan Strait to become the China mainland industrialization hatching station of Taiwan's traditional Chinese medicine products. Fourthly, both sides should actively carry out the method research of the quality control of cross-Strait Chinese crude drugs and decoction pieces, and the famous-region genuineness of cross-Strait CHMs, and the content analysis of active ingredients, and improve the value of application. Finally, China mainland especially Fujian and Taiwan should optimize and integrate the resources in new drug exploitation and marketing sufficiently, increase the R\&D investment to Chinese herbal drugs, make the technological research findings transform into the productivity quickly and create proper economic effectiveness and social benefit.

\subsection{Try to Unify the Standard, Management and Policy in TCMP}

Firstly, the different comprehension of the concept of “disease”, “syndrome”, “sign”, “disease entity”, “disease type”, "name of TCM syndrome” between Taiwan and China mainland, always causes some disturbance in the communication and cooperation of cross-Strait TCMP. The terminology of cross-Strait TCMP should be unified 
as early as possibly and also should be in line with international medicine educational circles. Secondly, the CHM species that have inconsistent origins also should be unified for the common standard, especially the species included in both Taiwan's and China mainland's pharmacopoeia. So the standard of pharmacopoeia should be unified too. Thirdly, China mainland and Taiwan District should establish the common overall borderland checkout mechanism to manage the export and import of Chinese crude drugs, and set up the report windows for intercommunication and coordinating mechanism to disqualified Chinese crude drug. Finally, the cross-Strait drug custody departments and industry associations should work together to explore the mutual recognition of cross-Strait standards and the co-operation of related testing technology. Aiming directly at the unconformity of quality standards for cross-Strait Chinese medicines, in accordance with the limited requirements of "Green Industry Standards for the Export and Import of Medicinal Plants and Preparation" to lead, cadmium, mercury, arsenic and other heavy metals, and the requirements of the 2010 edition "Pharmacopoeia of the People's Republic of China" to pesticide residues of organophosphates and pyrethroids, both sides should understand the quality control methods of cross-Strait Chinese herbal medicines and try to find a common method to unify the standard. The main focus is investigating the cross-Strait standards for Fujian bulk famous-region drugs, and comparing the standard differences, providing the technical support for the West Bank of Taiwan Strait to form the Taiwan export depot to Chinese herbal medicines. Ultimately through combining to enact the TCM pharmaceutical standards and the export of pharmaceutical certification, it will be as soon as possible to narrow the differences of crossStrait TCM management laws and regulations and quality standards to achieve mutual recognition.

\subsection{Strengthen the Safety Detection of Traditional Chinese Drugs}

Both sides should focus on the safety assessment of Chinese herbs. Before the export of Chinese crude drugs or traditional Chinese patent medicines and simple preparations in China mainland, we should strength the detection of toxic substances, harmful substances and heavy metals, moreover, take the same detected methods with Taiwan. To Taiwan's import, the detection also should be done like this.

\section{Conclusion}

To face the development and opportunities in the new history period, the cross-Strait medical academia and industry both generate the resonance from the academic to the industrial cooperation, give full play to their re- spective preponderance to jointly study and resolve the key issues that constraining the development of traditional Chinese medicine, and establish the long-term mechanism of cross-Strait TCMP cooperation. In order to make traditional Chinese drug modernize, the two sides of Taiwan Strait must be sharing the resources, have the mutual benefit and win-win to obtain collaborative progress, and make it widely to the international market. TCMP, passed down as one of the excellent treasures of the Chinese nation for safeguarding public health, its notable efficacy is extensively recognized by the people of China mainland and Taiwan District. Traditional Chinese medicine and pharmacy is the common wealth of the compatriots on both sides of the Taiwan Strait, and is also the important link to maintain the feelings of cross-Strait compatriots. To promote the prosperity and development of cross-Strait TCMP is the same aspiration of the Chinese people. The pharmaceutical industries in the both sides are willing to establish the combined research and development platform of Chinese medicine, so as to establish unified TCMP standards to improve the quality of varieties of cross-Strait Chinese medicine products and speed up their replacement, and improve the competitiveness and rapid, healthy development of cross-Strait TCMP industry.

\section{Acknowledgements}

The authors thank the Research Grant of Xiamen City Key Science and Technology Plan (No. 3502Z20100006) "Strait Science and Technology Platform (Xiamen, China) of Traditional Chinese Medicine and Pharmacy” to support this article.

\section{REFERENCES}

[1] X. C. Li, "Correlation between Quality Standard of Traditional Chinese Medicine Injections and Medication Safety," Chinese Journal of Pharmacoepidemiology, Vol. 21, No. 11, 2012, pp. 38-40.

[2] S. X. Wang, "The Scientization of Chinese Herbal Drugs and the New Drug Exploitation of Chinese Herbals Have Been Emphasized by Taiwan," Hebei Journal of Traditional Chinese Medicine, Vol. 25, No. 2, 2003, p. 113.

[3] Z. B. Yu, "From the Forum of Traditional Chinese Medicine and Pharmacy across Taiwan Strait to View the Trade Prospect of Traditional Chinese Drugs between Taiwan and Mainland China," Modern Chinese Medicine, Vol. 12, No. 7, 2010, pp. 42-43.

[4] Y. Huang, "The Progress and Tendency of Research of Traditional Chinese Medicine in Taiwan District," World Journal of Integrated Traditional and Western Medicine, Vol. 4, No. 6, 2009, pp. 452-454.

[5] "Six Cooperating Protocols Forming Complementation to Push about the Development of Traditional Chinese Medicine and Pharmacy in Taiwan District and China 
Mainland,”

http://www.chemdrug.com/databases/detail/3-13214.html 2010-07-08

[6] X. J. Gao, "10 Kinds of Chinese Crude Drugs Getting into Taiwan Will Be Put into Source Management and Needs to Provide the Inspection Unit Testimonial by China State Administration of Quality Supervision and Be Confirmed by Taiwan," Journal of Traditional Chinese Medicine Management, Vol. 19, No. 9, 2011, p. 859.

[7] T. B. Shih, "Research of System Analysis of Tonifying Chinese Herbal Medicines and Their Prescriptions Used on Human Stem Cells,” Yearbook of Chinese Medicine and Pharmacy, Vol. 24, No. 1, 2006, pp. 29-51.

[8] W. C. Wun. "Research on Processing Technique of Rhizoma Pinelliae (2-1)," Yearbook of Chinese Medicine and Pharmacy, Vol. 24, No. 3, 2006, pp. 1-39.

[9] S. S. Liou, “To Establish the Processing Standard and Processing Factory Norm of Chinese Crude Drug and Decoction Pieces of Cortex Magnoliae Officinalis,” Yearbook of Chinese Medicine and Pharmacy, Vol. 25, No. 4, 2007, pp. 89-146.

[10] L. R. Xiao, “The History Origin and Modern Development of Traditional Chinese Medicine in Fujian and Taiwan," Journal of Fujian University of Traditional Chinese Medicine, Vol. 16, No. 1, 2006, pp. 58-59.

[11] J. H. Guh, "Effect Research of Chinese Herbals' Ingredients on Antagonizing Cancer and Neovascularization and the Identification the Origin of Active Components (3 2)," Yearbook of Chinese Medicine and Pharmacy, Vol. 24, No. 2, 2006, pp. 75-94.

[12] M. D. Lin, “Taiwan Establishes the High-End Platform of Development and Verification of Traditional Chinese Medicine," China Medicine and Pharmacy, Vol. 1, No. 5, 2011, pp. 4-5.

[13] J. Z. Fu, "The New Change and Tendency of Traditional Chinese Medicine Education in Taiwan Recent Years," Journal of Traditional Chinese Medicine Management, Vol. 17, No. 8, 2009, pp. 686-688.

[14] X. Y. Su, "Current Situation of Higher Education of traditional Chinese Drug in Taiwan,” Chinese Journal of Information on Traditional Chinese Medicine, Vol. 14, No. 9, 2007, pp. 107-108.

[15] S. L. Huang, X. Y. Chen and C. M. Yang, "Play Area Advantage, Promote the Exchange and Development of Cross-Strait Traditional Chinese Medicine,” Strait Pharmaceutical Journal, Vol. 20, No. 10, 2008, pp. 142-145.

[16] N. Liao, “To Analyze the Cooperation and Development of Cross-Strait Industry of Chinese Medicine,” Journal of Traditional Chinese Medicine Management, Vol. 19, No. 6, 2011, pp. 497-500.

[17] X. F. Lin and D. Y. Lin, "General Situation of Administration of Traditional Chinese Medicine in Taiwan Area in 2009," Journal of Fujian University of Traditional Chinese Medicine, Vol. 21, No. 1, 2011, pp. 70-72.

[18] D. C. Tang, "Pre-Test on Education of under Graduate Course of Traditional Chinese Medicine in Taiwan," Lishizhen Medicine and Materia Medica Research, Vol. 17, No. 10, 2006, pp. 2096-2097.
[19] J. J. Shen, Y. L. Zhang and W. Q. Xu, "Present Situation and Suggestions of the Education of Chinese Medicine in Taiwan,” Education of Chinese Medicine, Vol. 21, No. 1, 2002, pp. 41-43.

[20] W. H. Bao, X. M. Liu, S. L. Ren and L. Li, "Compare the Setting Standards of TCM Hospitals in China Mainland with that in Taiwan District," World Chinese Medicine, Vol. 5, No. 6, 2010, pp. 436-439.

[21] Department of Planning and Finance of State Administration of Traditional Chinese Medicine, "China Statistical Yearbook of Chinese Medicine (1987-2010),” http://www.satcm.gov.cn/1987-2010/start.htm

[22] Department of Health, the Executive Yuan of Taiwan, "The Statistical Annual Report of Medical Institutions Status and Hospital's Utilization," Yearbook of Public Health of Taiwan, 2009, p. 23.

[23] B. W. Cheng, "Overview of Education of Professional Talented Person of Traditional Chinese Drug in Taiwan," Pharmaceutical Education, Vol. 11, No. 1, 1995, pp. 6061.

[24] L. Liu, "Many Points of View on Westernizing of Traditional Chinese Medical Hospitals,” Hospital Management Forum, Vol. 22, No. 11, 2005, pp. 36-40.

[25] X. H. Xiao, L. Q. Huang and X. J. Ma, "Discussion on the New Connotation and Significance of Traditional Chinese Drug and Its Modernization," China Journal of Chinese Materia Medica, Vol. 28, No. 3, 2003, pp. 282286.

[26] L. Jin, “Current Situation of Research and Development of Traditional Chinese Drugs and Thinking about the Future Development," China Medicine and Pharmacy, Vol. 1, No. 18, 2011, pp. 32-33.

[27] L. R. Xiao, "Characteristics, Research and Development of New Drugs of Traditional Chinese Medicine in Taiwan District for Recent Ten Years,” Journal of Fujian University of Traditional Chinese Medicine, Vol. 20, No. 2, 2010, pp. 64-65.

[28] G. Wang, J. Wang and W. Xu, "Study on the Extracting Condition of Antrodia Camphorata Polysaccharide,” Fujian Journal of Traditional Chinese Medicine, Vol. 42, No. 1, 2011, pp. 52-53.

[29] W. Xu, J. Wang and G. Wang, "Study on Toxicity Test for Antrodia Camphorata Capsule,” Strait Pharmaceutical Journal, Vol. 23, No. 5, 2011, pp. 41-43.

[30] L. H. Pan and W. Lu, "Analysis of Easy Confusion Species of Cross-Strait Traditional Chinese Drugs,” Journal of Fujian College of Traditional Chinese Medicine, Vol. 7, No. 1, 1997, pp. 33-34.

[31] D. Huang and L. L. Li, "Preliminary Discussion about Differences of TCM Quality Standards between Taiwan and Mainland China,” Chinese Pharmaceutical Affairs, Vol. 24, No. 11, 2010, pp. 1088-1090.

[32] D. S. Zhuang, P. Lin, Y. L. Zhang and L. Ye, "Strengthen the Technology Communication of Medical Science between Fujian and Taiwan to Promote the Development of Cross-Strait Health Service," Fujian Medical Journal, Vol. 17, No. 3, 1995, pp. 102-103.

[33] N. Liao, "Play Area Advantage and Carry forward Tradi- 
tional Culture: Fujian University of Traditional Chinese Medicine Paves the Way for the Bypass for the Exchange of Traditional Chinese Medicine between Fujian and Taiwan,” Relations across Taiwan Straits, Vol. 13, No. 3, 2009, pp. 52-53.
[34] M. Z. Lin, Z. B. Chen, "The Situation of Pharmaceutical Plant Resources in Southern Fujian,” Journal of Zhangzhou Normal University, Vol. 22, No. 4, 2009, pp. 99-103. 